\title{
Lowest uniformizations of compact Klein surfaces
}

\author{
Rubén A. Hidalgo
}

\begin{abstract}
A Schottky group is a purely loxodromic Kleinian group, with a nonempty region of discontinuity, isomorphic to a free group of finite rank. An extended Schottky group is an extended Kleinian group whose orientation-preserving half is a Schottky group. The collection of uniformizations of either a closed Riemann surface or a compact Klein surface is partially ordered. In the case of closed Riemann surfaces, the lowest uniformizations are provided by Schottky groups. In this paper we provide simple arguments to see that the lowest uniformizations of compact Klein surfaces are exactly those produced by extended Schottky groups.
\end{abstract}

\section{Introduction}

A compact Klein surface [1] is a connected smooth compact (with or without boundary) surface together with a maximal collection of smooth coordinate charts so that each transition function is either holomorphic or antiholomorphic. If all the transition functions are holomorphic and the boundary is empty, then the surface is a closed Riemann surface. A compact Klein surface that is not a closed Riemann surface is called a pure compact Klein surface. A pure compact Klein surface without boundary is called a pure closed Klein surface.

A dianalytic map between Klein surfaces is a continuous map which is, in each local chart, either holomorphic or antiholomorphic. A dianalytic automorphism of a compact Klein surface $S$ is a dianalytic self-homeomorphism; we denote by $\operatorname{Aut}(S)$ the group of dianalytic automorphisms of $S$. If $S$ is a Riemann surface, then every dianalytic automorphism is either a conformal or an anticonformal automorphism and, in this case, we denote by $\operatorname{Aut}^{+}(S)$ the group of conformal automorphisms.

There is an equivalence between the category of irreducible and nonsingular real projective algebraic curves and the category of pairs $\left(S^{+}, \tau\right)$, where $S^{+}$is a closed Riemann surface and $\tau: S^{+} \rightarrow S^{+}$is a symmetry (an anticonformal involution) of $S^{+}$. Also, if $S$ is a pure compact Klein surface, then there is a closed Riemann surface $S^{+}$and a symmetry $\tau: S^{+} \rightarrow S^{+}$so that $S$ and $S /\langle\tau\rangle$ are dianalytically

Mathematics Subject Classification (2010): 30F10, 30F40.

Keywords: Klein surfaces, uniformizations, Schottky groups, Kleinian groups. 
equivalent. The closed Riemann surface $S^{+}$is called a double oriented cover of $S$ and the genus of $S^{+}$is called the algebraic genus of $S$. All the above provides a natural equivalence between the category of pure compact Klein surfaces together with dianalytic maps, with the category of irreducible and nonsingular real projective algebraic curves together with real morphisms.

Let $S$ be a pure compact Klein surface, $S^{+}$a double oriented cover of $S$, $\tau: S^{+} \rightarrow S^{+}$a symmetry so that $S$ and $S^{+} /\langle\tau\rangle$ are dianalytically equivalent, and $P: S^{+} \rightarrow S$ a twofold (branched) cover with $\operatorname{deck}(P)=\langle\eta\rangle$. The map $P$ is unbranched if and only if $\eta$ has no fixed point ( $\eta$ is called an imaginary reflection). If $\eta$ has fixed points (in which case $\eta$ is called a reflection), then the locus of its fixed points consists of a collection of pairwise disjoint simple loops (called the ovals or mirrors of $\eta$ ). The image under $P$ of the fixed points of $\eta$ is the boundary of $S$, and the number of boundary components of $S$ is equal to the number of ovals of $\eta$.

A uniformization of a compact Klein surface $S$ is a triple $(\Delta, G, Q: \Delta \rightarrow S)$, where $\Delta$ is a planar Riemann surface, $G<\operatorname{Aut}(\Delta)$ acts properly discontinuously on $\Delta, G^{+}=G \cap \operatorname{Aut}^{+}(\Delta)$ (the orientation-preserving half of $G$ ) acts freely on $\Delta$ and $Q$ is a (branched) cover with $G$ as deck group. Notice that if $S$ is a closed Riemann surface, then $G=G^{+}$and $Q$ is unbranched. If $S$ is a pure compact Klein surface, then $G^{+} \neq G$ and $Q$ is unbranched if and only if $G$ contains no reflections.

Due to results of Haas [3] and Maskit [11], there is no loss of generality in assuming that $G$ is an extended Kleinian group with $\Delta$ as an invariant connected component of $G$ (see Section 2). The collection of the uniformizations of $S$ is partially ordered. If $S$ is a closed Riemann surface, then it is well known [12] that its lowest uniformizations are provided exactly by the Schottky uniformizations. If $S$ is a pure closed Klein surface, then in [5] we proved that the lowest uniformizatons are exactly those provided by extended Schottky groups (containing no reflections). In this paper we complete the story, that is, we provide simple arguments to see that the lowest uniformizations of pure compact Klein surfaces are exactly those provided by extended Schottky groups (see Theorem 1). This result may be of interest for studying algebraic real curves in terms of their uniformizations.

Theorem 1. The lowest uniformizations of pure compact Klein surfaces are exactly the extended Schottky ones, that is, the ones whose deck group is an extended Schottky group.

The proof of Theorem 1 is provided in Section 3. In Section 2 we recall some of the basic definitions and results we will need in the proof.

\section{Preliminaries}

We denote by $\widehat{\mathbb{M}}$ the group consisting of the Möbius transformations and the extended Möbius transformations (compositions of Möbius transformations with complex conjugation), and by $\mathbb{M}$ its index two subgroup of Möbius transformations. It is a well-known fact that $\operatorname{Aut}(\widehat{\mathbb{C}})=\widehat{\mathbb{M}}$ and that $\operatorname{Aut}^{+}(\widehat{\mathbb{C}})=\mathbb{M}$. 
If $G<\widehat{\mathbb{M}}$, then we set $G^{+}=G \cap \mathbb{M}$ (if $G$ contains extended Möbius transformations, then $G^{+}$is of index two in $G$ and it is called the orientation-preserving half of $G$ ).

\subsection{Extended Kleinian groups}

A Kleinian group (respectively, an extended Kleinian group) is a discrete subgroup of $\mathbb{M}$ (respectively, a discrete subgroup of $\widehat{\mathbb{M}}$ containing extended Möbius transformations). If $G$ is either a Kleinian group or an extended Kleinian group, then its region of discontinuity is the open subset (maybe empty) $\Omega(G) \subset \widehat{\mathbb{C}}$ of points on which $G$ acts discontinuously. If $K_{1}<K_{2}<\widehat{\mathbb{M}}$ and $K_{1}$ has finite index in $K_{2}$, then either one is a (extended) Kleinian group if and only if the other is; in which case both have the same region of discontinuity [9]. It follows that $G$ is an extended Kleinian group if and only if $G^{+}$is a Kleinian group; they share the same region of discontinuity.

A function group (respectively, an extended function group) is a pair $(G, \Delta)$, where $G$ is a finitely generated Kleinian group (respectively, finitely generated extended Kleinian group) and $\Delta \subset \Omega(G)$ is a $G$-invariant component of its region of discontinuity. Note that if $(G, \Delta)$ is an extended function group, then $\left(G^{+}, \Delta\right)$ is necessarily a function group, but the reciprocal is not true in general. We are interested in two particular classes of function groups, which we recall below.

A Schottky group of rank $g \geq 1$ is a Kleinian group $G<\mathbb{M}$ generated by loxodromic transformations $A_{1}, \ldots, A_{g}$, where there are $2 g$ disjoint simple loops, $C_{1}, C_{1}^{\prime}, \ldots, C_{g}, C_{g}^{\prime}$, with a common exterior $D$ in the Riemann sphere $\widehat{\mathbb{C}}$, where $A_{i}\left(C_{i}\right)=C_{i}^{\prime}$, and $A_{i}(D) \cap D=\emptyset, i=1, \ldots, g$. If $\Omega$ is the region of discontinuity of $G$, then it is well known that (i) $\Omega$ is a dense connected set, in particular, $(G, \Omega)$ is a function group, and (ii) $S=\Omega / G$ is a closed Riemann surface of genus $g$. A Schottky group of rank $g$ may be characterized as a finitely generated purely loxodromic free group of rank $g$ of Möbius transformations with nonempty region of discontinuity [10].

An extended Schottky group of rank $g$ is an extended Kleinian group for which its orientation-preserving half is a Schottky group of rank $g$; in particular, an extended function group. If $G$ is an extended Schottky group, then, as a Schottky group is torsion-free and has no parabolic transformations, every extended Möbius transformation $\tau \in G-G^{+}$is either a glide reflection (its square is a hyperbolic transformation) or an imaginary reflection (order two without fixed points on $\widehat{\mathbb{C}}$ ) or a reflection.

\subsection{Uniformizations}

A uniformization of a compact Klein surface $S$ is a triple $(\Delta, G, Q: \Delta \rightarrow S)$, where $\Delta$ is a planar Riemann surface, $G$ is a group of holomorphic and antiholomorphic automorphisms, acting properly discontinuously on $\Delta, G^{+}$(the orientation-preserving half of $G$ ) acts freely on $\Delta$ and $Q$ is a (branched) cover with $G$ as deck group. 
Theorem 2 (Haas [3], Maskit [11]). Let $\Delta$ be a planar Riemann surface and let $G=\operatorname{Aut}(\Delta)$. Then there is a conformal homeomorphism $h: \Delta \rightarrow \Omega$, where $\Omega$ is some open subset of $\widehat{\mathbb{C}}$, so that $h G h^{-1}<\widehat{\mathbb{M}}$.

As a consequence of Theorem 2, there is no loss of generality in assuming that if $(\Delta, G, Q: \Delta \rightarrow S)$ is a uniformization of a compact Klein surface $S$, then $G$ is a (extended) Kleinian group with $\Delta$ as an invariant connected component of $G$. For that reason, in what follows a uniformization of a closed Riemann surface $S$ will mean a triple $(\Delta, G, P: \Delta \rightarrow S)$, where $(G, \Delta)$ is a function group, $G$ acts freely on $\Delta, S=\Delta / G$ and $P: \Delta \rightarrow S$ is a holomorphic branched regular covering map with $G$ as deck group. If $G$ is a Schottky group, in which case $\Delta$ coincides with its region of discontinuity, the uniformization $(\Delta, G, P: \Delta \rightarrow S)$ is called a Schottky uniformization. As a consequence of the retrosection theorem, see for instance [2] and [8], every closed Riemann surface has Schottky uniformizations.

Similarly, a uniformization of a pure compact Klein surface $S$ will be a triple $(\Delta, G, P: \Delta \rightarrow S)$ where $(G, \Delta)$ is an extended function group, $G^{+}$acts freely on $\Delta, S=\Delta / G$ and $P: \Delta \rightarrow S$ is a dianalytic branched regular covering map with $G$ as deck group. In this case, the only elements of $G$ acting with fixed points on $\Delta$ are reflections (their locus of fixed points in $\Delta$ project to the boundary components of $S$ ). If $G$ is an extended Schottky group, then it is called an extended Schottky uniformization. It is not difficult to see, as consequence of quasiconformal deformation theory, that every pure compact Klein surface admits extended Schottky uniformizations.

The uniformizations of closed Riemann surfaces are described by the following result of B. Maskit:

Theorem 3 (Maskit [12]). Let $S$ be a closed Riemann surface.

(1) If $(\Delta, G, P: \Delta \rightarrow S)$ is a uniformization of $S$, then there exists a collection $\left\{\alpha_{m}\right\}$ of (homotopically independent) pairwise disjoint simple loops on $S$ and a collection of positive integers $\left\{k_{m}\right\}$ so that $P: \Delta \rightarrow S$ is a regular covering for which

a) each of the loops $w_{m}=\alpha_{m}^{k_{m}}$ lifts to a loop, and

b) every loop in $\Delta$ is freely homotopic to a product of liftings of the loops $w_{m}$.

(2) Reciprocally, given any collection $\left\{\alpha_{m}\right\}$ of (homotopically independent) pairwise disjoint simple loops on $S$ and a collection of positive integers $\left\{k_{m}\right\}$, there is a uniformization $(\Delta, G, P: \Delta \rightarrow S)$ of $S$ so that the regular covering $P: \Delta \rightarrow S$ satisfies a) and b) above. We say that the set of loops, $\left\{w_{m}=\alpha_{m}^{k_{m}}\right\}$, determines the uniformization $(\Delta, G, P: \Delta \rightarrow S)$ of $S$.

Given a uniformization $(\Omega, G, P: \Omega \rightarrow S)$ of a closed Riemann surface $S$ and some (holomorphic/antiholomorphic) homeomorphism $h: S \rightarrow S$, we say that $h$ lifts if there is some (holomorphic/antiholomorphic) homeomorphism $\widehat{h}: \Omega \rightarrow \Omega$ so that $h \circ P=P \circ \widehat{h}$. If $H$ is a group of (holomorphic/antiholomorphic) homeomorphisms of $S$, then we say that $H$ lifts if each $h \in H$ lifts. The next result provides necessary and sufficient conditions for a group $H$ to lift to a given uniformization. 
Theorem 4 ([7], [4]). Let $(\Omega, G, P: \Omega \rightarrow S)$ be a uniformization of a closed Riemann surface $S$ of genus $g \geq 2$ and let $H$ be a group of (holomorphic/antiholomorphic) automorphism of $S$. Then $H$ lifts if and only if there is a collection $\mathcal{F}$ of pairwise disjoint loops (powers of simple loops) on $S$, defining the covering $P: \Omega \rightarrow S$, which is invariant under the action of $H$.

We should note that if in Theorem 4 the group $G$ has torsion, then (by Theorem 3) some of the defining loops should be nontrivial powers of simple loops. There are also situations in which $G$ is torsion-free, but there are defining loops which are nontrivial powers of simple loops (this only happens in presence of accidental parabolic elements).

The set of uniformizations of a compact Klein surface $S$ is partially ordered as follows. We say that a uniformization $\left(\Delta_{1}, K_{1}, P_{1}: \Delta_{1} \rightarrow S\right)$ is higher than a uniformization $\left(\Delta_{2}, K_{2}, P_{2}: \Delta_{2} \rightarrow S\right)$ if there is a holomorphic map (necessarily a covering) $Q: \Delta_{1} \rightarrow \Delta_{2}$ so that $P_{1}=P_{2} \circ Q$. Equivalently, if there exists a normal subgroup $L$ of $K_{1}$ so that $K_{2}=K_{1} / L$ (in which case $L$ is the deck group of the covering $Q)$.

The highest uniformizations $(\Delta, K, P: \Delta \rightarrow S)$ of $S$ correspond to the case when $\Delta$ is simply connected, for instance, by (i) Fuchsian groups in the case of closed Riemann surfaces of genus at least two, and (ii) by non-Euclidean crystallographic (NEC) groups for the case of pure compact Klein surfaces of algebraic genus at least two.

It follows from the definition that a uniformization $(\Delta, K, P: \Delta \rightarrow S)$ is a lowest one if there is no nontrivial normal subgroup $N \triangleleft K$, with $\Delta / N$ a planar Riemann surface. Notice that in this case necessarily $N \triangleleft K^{+}$. It is well known that the lowest uniformizations of closed Riemann surfaces are provided by the Schottky ones [12].

If $S$ is a pure compact Klein surfaces and $(\Delta, G, P: \Delta \rightarrow S)$ is a uniformization, then there is a uniformization $\left(\Delta, G^{+}, P^{+}: \Omega \rightarrow S^{+}\right)$and a twofold branched cover $Q: S^{+} \rightarrow S$ so that $P=Q \circ P^{+}$. As Schottky groups provide the lowest uniformizations of closed Riemann surfaces, it follows that extended Schottky uniformizations are necessarily lowest ones. The converse, for the case of pure compact unbordered Klein surfaces of algebraic genus at least two, was obtained in [5]. Theorem 1 extends this to all pure compact Klein surfaces.

Remark 5. In [6] we have used a different partial ordering on the collection of uniformizations, in which case the lowest uniformizations are different from the ones considered here. Let us first recall the partial order used in that paper. Assume we have a uniformization $(\Delta, K, P: \Delta \rightarrow S)$ of a pure compact Klein surface $S$. Set $S^{0}=S-\partial S$, where $\partial S$ is the boundary of $S$. Choose a connected component of $P^{-1}\left(S^{0}\right)$, say $\widehat{\Delta}$. In this way, we get a regular planar covering $P: \widehat{\Delta} \rightarrow S^{0}$. Different choices provide isomorphic regular coverings of $S^{0}$. We may now induce a partial order on the collection of uniformizations in this setting as follows. Let us consider uniformizations $\left(\Delta_{1}, K_{1}, P_{1}: \Delta_{1} \rightarrow S\right)$ and $\left(\Delta_{2}, K_{2}, P_{2}: \Delta_{2} \rightarrow S\right)$. As before, we consider regular coverings $P_{1}: \widehat{\Delta}_{1} \rightarrow S^{0}$ and $P_{2}: \widehat{\Delta}_{2} \rightarrow S^{0}$. Then we say that the uniformization $\left(\Delta_{1}, K_{1}, P_{1}: \Delta_{1} \rightarrow S\right)$ is higher than $\left(\Delta_{2}, K_{2}, P_{2}: \Delta_{2} \rightarrow S\right)$ 
if there is a holomorphic map (necessarily a covering) $Q: \widehat{\Delta}_{1} \rightarrow \widehat{\Delta}_{2}$ so that $P_{1}=$ $P_{2} \circ Q$. Using this partial ordering, we proved in [6] that the lowest uniformizations $(\Delta, K, P: \Delta \rightarrow S)$ are those for which $K$ is constructed from Klein-Maskit's free combination of cyclic groups; a very particular class of extended Schottky groups. For instance, the extended Schottky group $\Gamma=\langle A(z)=2 z, B(z)=\bar{z}\rangle$ does not belong to this class of extended Schottky groups. The region of discontinuity of $\Gamma$ is $\Delta=\mathbb{C}-\{0\}$ and $S=\Delta / \Gamma$ is topologically a closed annulus. Let $P: \Delta \rightarrow S$ a (branched) regular covering with $\Gamma$ as deck group. So $(\Delta, \Gamma, P: \Delta \rightarrow S)$ is a uniformization of $S$ and, moreover, $\Delta-P^{-1}(\partial S)=\widehat{\Delta}^{+} \cup \widehat{\Delta}^{-}$, where $\widehat{\Delta}^{+}=\{z \in \mathbb{C}$ : $\operatorname{Im}(z)>0\}$ and $\widehat{\Delta}^{+}=\{z \in \mathbb{C}: \operatorname{Im}(z)<0\}$. We may choose $\widehat{\Delta}=\widehat{\Delta}^{+}$. In this case, $P: \widehat{\Delta}^{+} \rightarrow S-\partial S$ is a universal covering which clearly will not be a lowest one for the given definition. In fact, a lowest uniformization of $S$ in this case is provided by the group generated by the reflections through two disjoint circles.

\section{Proof of Theorem 1}

Let $S$ be a pure compact Klein surface of algebraic genus $g$. The particular cases $g \in\{0,1\}$ are not hard to check (we leave the details to the interested reader). So, henceforth, we assume that $g \geq 2$. We have already noticed that extended Schottky groups provide lowest uniformizations of compact Klein surfaces. We proceed to prove the converse, that is, every lowest uniformization of a compact Klein surface is an extended Schottky one.

Let $(\Omega, K, P: \Omega \rightarrow S)$ be a lowest uniformization of $S$ and set $S^{+}=\Omega / K^{+}$(a closed Riemann surface of genus $g \geq 2)$. We have the uniformization $\left(\Omega, K^{+}, Q\right.$ : $\left.\Omega \rightarrow S^{+}\right)$of $S^{+}$. Let $\eta: S^{+} \rightarrow S^{+}$be the symmetry induced by any $\tau \in K-K^{+}$; so $S=S^{+} /\langle\eta\rangle$. Let $T: S^{+} \rightarrow S$ be a (possibly branched) twofold covering with deck group $\langle\eta\rangle$ so that $T \circ Q=P$. As consequence of Theorem 4 we have a collection $\mathcal{F}$ of pairwise disjoint loops on $S^{+}$which is invariant under the action of $\eta$ and defines the regular planar covering $Q: \Omega \rightarrow S^{+}$.

We claim that $K^{+}$is torsion-free. In fact, let $\mathcal{G}$ be the collection of pairwise disjoint simple loops on $S^{+}$for which a power of the loop belongs to $\mathcal{F}$. The collection $\mathcal{G}$ defines a holomorphic regular planar covering $Q_{1}: \Omega_{1} \rightarrow S^{+}$so that there is a planar covering $Q_{2}: \Omega \rightarrow \Omega_{1}$ with $Q=Q_{1} \circ Q_{2}$. As $\eta$ keeps invariant the collection $\mathcal{G}$, we have that $\eta$ lifts, with respect to the cover $Q_{1}: \Omega_{1} \rightarrow S^{+}$, to an antiholomorphic automorphism of $\Omega_{1}$. By Theorem 3, we may assume the deck group of this covering to be given by some function group $(G, \Delta)$ with $\Omega_{1}$ contained in $\Delta$ and that $\widehat{\eta} \in \widehat{\mathbb{M}}$. As $\Omega_{1} / G=S^{+}$is compact, we must have $\Omega_{1}=\Delta$. Now, $T \circ Q_{1}: \Omega_{1} \rightarrow S$ defines a regular planar covering of $S$. In order to avoid a contradiction with the minimality of the uniformization $(\Omega, K, P: \Omega \rightarrow S)$, we must have that $Q_{2}$ is a homeomorphism, that is, $\mathcal{F}=\mathcal{G}$ and, by Theorem 3 , that $K^{+}$is torsion-free.

As a consequence, there is a collection $\mathcal{F}$ of pairwise disjoint simple loops on $S^{+}$ invariant under the action of $\eta$ defining the regular planar covering $Q: \Omega \rightarrow S^{+}$.

In order to finish the proof, we only need to prove that $\left(\Omega, K^{+}, Q: \Omega \rightarrow S^{+}\right)$ is a lowest uniformization, as in this case, we know that $K^{+}$should be a Schottky group and, in particular, that $K$ is an extended Schottky group as desired. 
If $\left(\Omega, K^{+}, Q: \Omega \rightarrow S^{+}\right)$is not a lowest uniformization, then one of the connected components of $S^{+}-\mathcal{F}$ should have positive genus. Let us denote such a component by $X$. We have that either $\eta(X)=X$ or that there is another connected component, say $Y$, with $\eta(X)=Y$. If $\eta(X)=X$, then we may construct a homotopically nontrivial simple loop $\delta \subset X$, so that $\eta(\delta)=\delta$. In the case that $\eta(X)=Y$ we may consider any homotopically nontrivial simple loop $\gamma \subset X$. By adding $\delta$ (if $\eta(X)=X$ ) or by adding $\gamma$ and $\eta(\gamma)$ (if $\eta(X)=Y$ ) to the collection $\mathcal{F}$, we obtain a larger collection $\mathcal{N}$ of pairwise disjoint simple loops which is invariant under $\eta$. Moreover, by the choice of the new loops loops ( $\delta$ in the first case; $\gamma$ and $\eta(\gamma)$ in the second case), we see that the uniformzation induced by $\mathcal{N}$ is a lowest one than $(\Omega, K, P: \Omega \rightarrow S)$, a contradiction.

\section{References}

[1] Alling, N. L. And Greenleaf, N.: Foundations of the theory of Klein surfaces. Lecture Notes in Math. 219, Springer-Verlag, Berlin-New York, 1971.

[2] Bers, L.: Automorphic forms for Schottky groups. Adv. in Math. 16 (1975), $332-361$.

[3] HaAs, A.: Linearization and mappings onto pseudocircle domains. Trans. Amer. Math. Soc. 282 (1984), no. 1, 415-429.

[4] Hidalgo, R. A.: Automorphisms groups of Schottky type. Ann. Acad. Sci. Fenn. 30 (2005), 183-204.

[5] Hidalgo, R. A. and Maskit, B.: On Klein-Schottky groups. Pacific J. Math. 220 (2005), no. 2, 313-328.

[6] Hidalgo, R. A. And Maskit, B.: Lowest uniformizations of compact real surfaces. Contemporary Math. 397 (2006), 145-152.

[7] Hidalgo, R. A. And Maskit, B.: A note on the lifting of automorphisms. In Geometry of Riemann surfaces, 260-267. London Math. Soc. Lecture Note Ser. 368, Cambridge Univ. Press, Cambridge, 2010.

[8] Koebe, P.: Über die Uniformisierung der Algebraischen Kurven II. Math. Ann. 69 (1910), 1-81.

[9] Maskit, B.: Kleinian groups. Grundlehren der Mathematischen Wissenschaften 287, Springer-Verlag, Berlin, 1988.

[10] Maskit, B.: A characterization of Schottky groups. J. Anal. Math. 19 (1967), $227-230$.

[11] Maskit, B.: The conformal group of a plane domain. Amer. J. Math. 90 (1968), no. 3, 718-722.

[12] Maskit, B.: A theorem on planar covering surfaces with applications to 3-manifolds. Ann. of Math. 81 (1965), no. 2, 341-355.

Received June 16, 2011; revised January 3, 2012.

Rubén A. Hidalgo: Departamento de Matemática, Universidad Técnica Federico Santa María, Casilla 110-V Valparaiso, Chile.

E-mail: ruben.hidalgo@usm.cl 